\title{
THE PHOTOGRAPHIC METHOD FOR RECORDING AVERAGE ILLUMINATIONS
}

\author{
By L. E. Bayliss, B.A., Ph.D. \\ Department of Physiology, University of Edinburgh, and the \\ Marine Biological Laboratory, Plymouth
}

(Text-figs. I-3)

The photo-electric cell is admirably suited for measuring instantaneous illuminations, but does not lend itself readily to the measurement of the total illumination received on a surface over a long period of time, or the average illumination received during that time, although it has been used for this purpose (Atkins \& Poole, I936a). The method used by these authors, however, would be difficult to use in the field and away from laboratory facilities. The photographic method, on the other hand, is admittedly not capable of yielding results of high precision, but the simplicity of operation, and absence of elaborate and bulky apparatus, make it suitable for use in the field.

In principle, the amount of blackening produced in a photo-sensitive emulsion is used as a measure of the total illumination received; in order to increase the range over which measurements can be made, a neutral tint wedge, of suitable slope, is placed in front of the emulsion, and the distance along the wedge at which a standard amount of blackening is produced is taken as a measure of the illumination. The standard amount of blackening is best defined as that amount which is produced, in the absence of the wedge, by a known illumination acting for a known time. The method has inherent limitations, particularly when used for long-period observations, which result from $(a)$ the failure of the reciprocity law, $(b)$ the uncertainty in the value of the wedge constant, and $(c)$ the varying sensitivity of the emulsion to lights of different wave-lengths.

The use of the neutral tint wedge in photometry has been fully described by Hecht (I9I8) and Eder (I9I9, I920a, b). These authors, however, do not appear to have considered in any detail the possible effects of the limitations just mentioned, although their existence is recognized. Gruber (1924) and Oberdorfer (1928) used the EderHecht photometer for ascertaining the intensity of illumination at various depths below the surface of a lake, and Cooper \& Sayce (I932) made continuous records of the intensity of daylight throughout the day, with the aid of a neutral tint wedge. Such observations, however, fail to make use of the innate integrating properties of the photographic emulsion.

Let the emulsion be exposed behind the wedge to a constant illumination $I$ for a time $t$. Let the print of the wedge at a distance $l$ from the zero end of the wedge have the same density (amount of blackening) as that produced by the 
standardizing light, of intensity $I_{s}$ acting for the same time, $t$. Then the intensity of light actually reaching the emulsion must be the same during both exposures. Now, if $I^{\star}$ is the intensity of light emerging from the wedge, and striking the emulsion, we can write, from the known properties of the wedge,

$$
\log I^{\star}=\log I-k l,
$$

where $k$ is the "wedge constant". Hence, in the particular case considered,

$$
\log I=\log I_{s}+k l \text {. }
$$

If, however, the duration of the standardizing exposure is not the same as that of the wedge exposure, or if the intensity of the illumination during the latter is not constant, complications arise.

\section{The Failure of the Reciprocity LaW}

The total illumination received during any time $t$ is given by $R=I t$, if the intensity is constant. The amount of blackening produced in a photographic emulsion, however, by a constant illumination acting for a time, $t$, does not, in general, follow this "reciprocity law", but depends upon the product $I t^{p}$, where $p$ is the "Schwarzschild constant" (Schwarzschild, I900). If, as before, $I^{\star}$ is the illumination actually reaching the emulsion, during the wedge exposure, at the point $l$, as previously defined,

$$
R^{\star}=I^{\star} t \text {, }
$$

whence

$$
\log R=\log R^{\star}+k l \text {. }
$$

But at the point $l$ the amount of blackening produced under the wedge is the same as that produced by the standardizing exposure of intensity $I_{s}$ and of duration $t_{s}$. We can write, therefore,

or

$$
\begin{aligned}
I^{\star} t^{p} & =I_{s} t_{s}{ }^{p} \\
R^{\star} t^{p-1} & =R_{s} t_{s}^{p-1},
\end{aligned}
$$

whence

$$
\log R=\log R_{s}+k l+(p-\mathrm{I}) \log \left(t_{s} / t\right) .
$$

An extensive treatment of the law of blackening of the photographic emulsion, and the failure of the reciprocity law, is given in the monograph by Dobson, Griffith \& Harrison (I926).

Clearly, then, a correction must be introduced whenever the standardizing exposure and the wedge exposure are of different durations, unless the value of $p$ approximates sufficiently closely to unity.

Table I. Correction to be applied to Photographic Records due to DEPARTURE FROM THE RECIPROCITY LAW

\begin{tabular}{cccccc} 
& \multicolumn{5}{c}{$t_{s} / t$} \\
$p$ & $\overbrace{1 . I}$ & 0.2 & 0.3 & 0.5 & 0.7 \\
$I .03$ & 0.935 & 0.95 & 0.965 & 0.980 & 0.990 \\
I.05 & 0.890 & 0.92 & 0.935 & 0.965 & 0.980 \\
I.IO & 0.790 & 0.85 & 0.885 & 0.930 & 0.965
\end{tabular}


Values of this correction are given in Table I. Two points are of significance in connexion with these figures. (a) In many cases, the total illumination received over the whole period of daylight must be measured, while it is inconvenient to continue the standardizing exposure for more than I or $2 \mathrm{hr}$. (b) In such cases, the exact duration of the illumination under measurement is difficult, or impossible, to discover; this is usually of no importance, except that the exact value of the correction to be applied is also unknown, and so must be kept small. It will be shown in a later section that the inherent experimental uncertainty of the method is about $\pm 10 \%$. The maximum tolerable departure of the value of $p$ from unity is thus about \pm 0.05 .

Non-uniform illumination. The magnitude of the correction necessary when the illumination is fluctuating depends, obviously, on the nature of the fluctuation. Two special cases, however, may be considered.

(I) The exposure is made on a clear day from sunrise to sunset, so that we may write, approximately,

$$
I=I_{0} \sin \frac{\pi t}{12},
$$

where $t$ is the time in hours. The total radiation received is thus

$$
R=\int_{0}^{12} I_{0} \sin \frac{\pi t}{\mathrm{I} 2} d t=\frac{24}{\pi} I_{0} .
$$

The amount of blackening produced, however, depends upon the quantity $S$, where

$$
S=\int_{0}^{12}\left(\begin{array}{l}
\partial S \\
\partial t
\end{array}\right) d t=\int_{0}^{12} p t^{p-1} I_{0} \sin \frac{\pi}{\mathrm{I} 2} t d t .
$$

Graphic integration of this expression, putting $p=\mathrm{I} \cdot \mathrm{IO}$, gives

$$
S=\text { IO I } I_{0} \text {. }
$$

We will assume, for simplicity, that the standardizing exposure has the same duration as the wedge exposure, hence

$$
S_{s}=(\mathrm{I} 2)^{1 \cdot 10} I_{s}=\mathrm{I} 5.4 I_{s} \text {. }
$$

Similarly

$$
\begin{aligned}
R & =\frac{24}{\pi} I_{0}, \\
R_{s} & =\mathrm{I} 2 I_{s} .
\end{aligned}
$$

If the amount of blackening produced in the two exposures is the same, then $S=S_{s}$, and

$$
I_{0}=\frac{\mathrm{I} 5 \cdot 4}{\mathrm{IO} \cdot \mathrm{I}} I_{s}=\mathrm{I} \cdot 53 I_{s} .
$$

The fact that the unknown exposure produced the same amount of blackening 
as the standardizing exposure, however, would lead us to suppose that the total radiation received in both cases was the same, i.e. that

$$
R_{s}=\mathrm{I} 2 I_{s}=R_{0}=\frac{24}{\pi} I_{0}
$$

or that

$$
I_{0}=\mathrm{I} \cdot 57 I_{s} \text {. }
$$

The estimate of the total illumination received, in terms of the amount of blackening produced, will thus be too high by about $3 \%$.

(2) The illumination is fluctuating in a simple harmonic fashion about a fixed value. This approximates to the conditions in the neighbourhood of noon, with intermittent clouds. The intensity at any moment can now be written,

$$
I=I_{1}+I_{2} \sin \omega t .
$$

Since the greatest fluctuations, and hence the greatest possible discrepancy, will be when the illumination falls periodically to zero, we can simplify the expression, and write

and

$$
\begin{aligned}
& I=I_{0}(\mathrm{I}+\sin \omega t) \\
& R=I_{0} \int_{0}^{t}(\mathrm{I}+\sin \omega t) d t .
\end{aligned}
$$

If $t$ is such as to occupy any whole number of waves, this reduces to $R=I_{0} t$, i.e. the fluctuations do not affect the total illumination received.

Similarly

$$
\begin{aligned}
S & =p I_{0} \int_{0}^{t} t^{p-1}(\mathrm{I}+\sin \omega t) d t \\
& =I_{0} t^{p}+p I_{0} \int_{0}^{t} t^{p-1} \sin \omega t d t .
\end{aligned}
$$

The second term in this expression has the property that the area of any halfwave is greater than the area of the preceding half-wave. The area of successive whole waves, however, approaches zero as $t$ becomes greater, so that the area of the first whole wave will give a measure of the maximum discrepancy introduced, which will not be exceeded however many subsequent waves are included in addition. Since, in general, any type of fluctuation can be reduced to a series of simple harmonic waves of suitable frequency, this discrepancy must also be the maximum which will be introduced by any type of fluctuation about a finite mean.

Graphic integration, with $p=\mathrm{I} \cdot \mathrm{IO}$ as before, over the first complete wave gives $I_{0}=\mathrm{I} \cdot 04 I_{s}$ as the condition that the same amount of blackening shall be produced by the fluctuating light as by the standardizing light. We should be led to believe, however, that $I_{0}=I_{s}$, and the discrepancy introduced is thus about $4 \%$. The photographic measure of the illumination will be smaller or greater than the true measure according as the exposure was begun while the illumination was rising or falling. 
In general, then, fluctuations in the illumination will not introduce any discrepancy greater than 2 or $3 \%$ so long as the Schwarzschild constant does not differ from I by more than $0 \cdot 10$.

\section{The Wedge Constant}

The wedge was of the Goldberg (I910) pattern and was supplied by Messrs Ilford, Ltd. It was $2 \mathrm{I} \mathrm{cm}$. long by I.25 cm. wide, with a density of $3 \cdot 0$ at the dense end and was cut in the middle, the two halves being mounted side by side so as to form a rectangle $10.5 \times 2.5 \mathrm{~cm}$.; a wide range of densities was thus available in a compact form. The wedge itself was mounted between two pieces of glass, one of which was made as thin as possible ( $\mathrm{I} \mathrm{mm}$.) in order to reduce the shading effect of the edges, and the errors introduced by parallax between the wedge and the paper.

The makers of the wedge furnish a figure for the relation between the density of the wedge at any point, and the distance of this point from the end of the wedge; this is derived from measurements made in the photometer described by Benson, Ferguson \& Renwick (I9I8) and will not necessarily be applicable in all circumstances. The disturbing factors are (I) the inclination of the incident light to the normal, and (2) the wave-length sensitivity of the photo-sensitive material with which the wedge is used. The first arises from the fact that a ray of light passing through the wedge obliquely has to traverse a greater thickness of absorbing material than one passing through normally. The second arises from the fact that the material of which the wedge is composed, while strictly "neutral" in that the density at any point is independent of the wave-length for all values greater than about $420 \mathrm{~m} \mu$, becomes rapidly denser with decrease in wave-length below this figure, as has been shown by Toy \& Ghosh (1920). In the Ferguson-Benson-Renwick photometer, the wedge is illuminated by diffuse light and the densities are measured visually, so that the effective wave-length is considerably larger than $420 \mathrm{~m} \mu$.

\section{The EfFect of the InCLination of THE INCIDENT Light}

If the wedge is illuminated by light derived from a diffusing surface, which is itself illuminated by approximately parallel light incident nearly normally, the conditions will be identical with those in which it is calibrated, and the makers' figure may be used. It is of interest, however, to investigate what corrections would be needed in the following circumstances. (I) The degree of imperfection of the diffusing surface used differs from that of the diffuser used by the makers (a "perfect" diffusing surface is considered to be one from which the illumination emitted per unit solid angle is independent of the direction of emission, i.e. the illumination emitted per unit area is proportional to the cosine of the angle of emission). (2) Parallel light is incident obliquely on an imperfect diffusing surface. (3) Parallel light is incident 
obliquely on the wedge without interposition of a diffusing surface. (4) The wedge is illuminated by the system of two lamps and diffusing surface used in the measurement of the Schwarzschild constant (see below).

(I) Calculation of the ratio of the wedge constant in diffuse light to that in parallel light. Let the wedge be illuminated by an infinite diffusing surface. Consider the illumination received by any point on the wedge from an elementary annulus which subtends an angle $\theta$ at this point. Let the illumination emitted per unit solid angle be $I_{\theta} . I_{\theta}$ is a measure of the imperfection of the diffusing surface, and is independent of $\theta$ if the illumination is perfectly diffuse. Then, the illumination emitted per unit surface in the direction $\theta$ is $I_{\theta} \cos \theta$.

The total illumination received at the point considered from the elementary annulus is $2 \pi I_{\theta} \sin \theta d \theta$, and the vertical component is $2 \pi I_{\theta} \sin \theta \cos \theta d \theta$. The total illumination falling on the wedge $=I=\int_{0}^{\pi} 2 \pi I_{\theta} \sin \theta \cos \theta d \theta$. This expression reduces to $\pi I_{\theta}$ for perfectly diffuse light, and can be evaluated graphically if the relation between $I_{\theta}$ and $\theta$ is determined empirically for any given diffusing surface. But this illumination passes through the wedge at an angle $\phi$, where $\sin \theta / \sin \phi=\mu$, the refractive index of the material of which the wedge is composed. Hence, if $D$ is the density of the wedge at the point considered to light of normal incidence, the density to light incident at an angle $\theta$ is $D / \cos \phi$, and the vertical component transmitted is

$$
\frac{2 \pi I_{\theta} \sin \theta \cos \theta d \theta}{(\mathrm{IO})^{D / \cos \phi}} .
$$

The total illumination received on the paper after passing through the wedge is thus

$$
\int_{0}^{\pi} \frac{2 \pi I_{\theta} \sin \theta \cos \theta d \theta}{(\mathrm{IO})^{D / \cos \phi}}=I^{\star} .
$$

Integration can again be carried out graphically. The effective density of the wedge to light emitted from any given diffusing surface is given by $\log _{10} I^{\star} / I=D^{\prime}$. For perfectly diffuse light, the ratio $D^{\prime} / D$ was calculated to be I.I35, while for light from two samples of surface-flashed opal glass, the ratio $D^{\prime} / D$ was found to be I.I2O and I.I25 respectively; the second of these was markedly more nearly "perfect" than the first. $\mu$ has been taken as $I^{\cdot} 5$, a value which is probably rather too large.

The "imperfection" of an actual diffuser was measured by projecting an image of an incandescent filament $2 \mathrm{~mm}$. wide normally on one side; the intensity of the light emitted on the far side at a number of angles to the normal was then measured by means of a rectifier photo-cell behind a slit $2 \mathrm{~mm}$. wide, and $45 \mathrm{~mm}$. from the diffuser. The photo-cell and galvanometer used were calibrated by means of an inverse square law photometer.

It would appear that variations in the "perfection" of the diffuser over reasonable limits will not seriously affect the value of the wedge constant. 
Atkins \& Poole ( $1936 b)$ remark that "a I mm. filter is about equivalent to a similar one I. $15 \mathrm{~mm}$. thick, if it is used in diffuse light". The calculated figures are in adequate agreement with this observation.

(2) The effect of parallel light incident obliquely on an imperfect diffuser. In this case, the illumination emitted by the elementary annulus will not be uniform at all points, but will reach a maximum in the direction of the incident light. The distribution of intensity of the light emitted was measured by projecting the image of the incandescent filament on the diffusing surface at an angle to the normal, either in the plane of rotation of the photo-cell, or in a plane at right angles. From the approximate distribution curve so obtained, the mean circular intensity was derived, and used as the value of $I_{\theta}$ in the expression given in the previous section. For an angle of incidence of $60^{\circ}$, and the less perfect diffusing surface, the value of $D^{\prime} / D$ so obtained was I.I4. The distribution curve was very little affected by further increase in the angle of incidence, so that we may take it that for this particular diffusing surface, the value of $D^{\prime} / D$ would lie between I.I2 and I.I4 for all angles of incidence. Further, the figures are not likely to be altered significantly by considerable changes in the degree of imperfection of the diffusing surface.

(3) Parallel light is incident obliquely on the wedge without the interposition of a diffusing surface. Here, $D^{\prime}=D / \cos \phi$, where $\sin \theta / \sin \phi=\mu$. If $\mu$ is taken as $\mathrm{I}^{\cdot} 5$, the values given in Table II are obtained. In actual practice, however,

\section{Table II. Relative Values of Wedge Constant in Lights OF VARYING OBLIQUITY}

$\begin{array}{lccccc}\text { Angle of incidence } & 0^{\circ} & 30^{\circ} & 45^{\circ} & 60^{\circ} & 90^{\circ} \\ D^{\prime} / D \text { calc. } & \mathrm{I} \cdot 00 & \mathrm{I} \cdot 06 & \mathrm{I} \cdot \mathrm{I} 35 & \mathrm{I} \cdot 22 & \mathrm{I} \cdot 34 \\ D^{\prime} / D \text { daylight conditions } & - & \mathrm{I} \cdot \mathrm{rO} & \mathrm{I} \cdot \mathrm{I} 35 & \mathrm{I} \cdot \mathrm{I} 6 & -\end{array}$

if a wedge is exposed to bright sunlight, the value of $D^{\prime} / D$ will not vary to so great an extent with the altitude of the sun. In the first place, there is a considerable amount of scattering of light within the wedge itself, with the result that the observed value of $D^{\prime} / D$ for an imperfect diffuser was $I^{\cdot} \cdot 09 \pm 0.02$ instead of the calculated I.I2. In the second place, the wedge is never exposed to pure sunlight, but to a mixture of sunlight and diffuse skylight. Atkins \& Poole (1936a) give a large number of values for the ratio of the vertical component of sunlight to the vertical component of skylight, from which average values can be obtained for various altitudes of the sun. The estimated actual values of $D^{\prime} / D$ for sunlight + daylight are given in the last line in Table II. In addition, if a wedge recorder is exposed at sea, the random motion will largely average out the values of $D^{\prime} / D$. The average of the figures for pure sunlight given above is $\mathrm{I} \cdot \mathrm{I} 5$, a value which differs little from that for diffuse light.

A second-order effect on the wedge constant produced by light incident obliquely in the plane of the long axis of the wedge arises from the fact that light reaching the photo-sensitive surface at a point distant $l$ from the zero end of the wedge, will pass 
through the wedge at some point distant $l \pm \Delta l$ from the zero end. If $d$ is the thickness of the glass between the wedge and the photo-sensitive surface, $\Delta l=d \tan \phi$. Now the maximum value of $\phi$ is $\sin ^{-1} \mu$, where $\mu$ is the refractive index of the glass. If this is taken as $\mathrm{I} \cdot 5$, the maximum value of $\Delta l$, for $d=\mathrm{I} \mathrm{mm}$., becomes $0.9 \mathrm{~mm}$., corresponding to a maximum increase or decrease in density of $3 \%$. This effect, of course, will not be apparent in diffuse light, since the positive and negative corrections will be equal.

It is clear, therefore, that it is always advisable to place a sheet of diffusing material above the wedge. The extra uncertainty introduced by the omission of the diffuser, however, is not likely to be large except when the recorder is exposed on land to bright sunlight with the altitude of the sun greater than about $55^{\circ}$ or less than about $35^{\circ}$. If the diffuser is used, care must be taken that the diffusing surface is large enough to allow the intensity of illumination at the ends of the wedge to be the same as that in the centre. It can easily be shown that the necessary minimum length of a perfect diffusing surface increases as its distance from the wedge increases. If the wedge is $10 \mathrm{~cm}$. long, and the diffusing surface is $5 \mathrm{~cm}$. from it, and $25 \mathrm{~cm}$. long, the intensity at the ends of the wedge will be $3 \%$ less than that at the centre. If, on the other hand, the diffusing surface is only $0.5 \mathrm{~cm}$. from the wedge, a $3 \%$ difference of intensity will be obtained with a diffusing surface only II cm. long. In both cases the illumination sensibly reaches the value at the centre at points $0.5 \mathrm{~cm}$. from the ends.

It may be pointed out that any uncertainty in the value of the wedge constant only becomes significant if the illumination integrals to be compared differ considerably in magnitude. Thus while it may not be possible to determine with great accuracy the absolute value of, say, the total illumination received during one day, it is possible to compare this illumination with that received during another day with considerable accuracy.

(4) The constant of the wedge when exposed to the source of light used for the measurement of the Schwarzschild constant. The value was found by direct measurements to be identical with that for diffuse light, except when measurements were made at points immediately opposite one of the lamps. As was to be anticipated, owing to the imperfection of the diffusing arrangements, the wedge constant at these points was lower than at other points-actually by about $3.5 \%$. This discrepancy is of no consequence, as the extreme ends of the wedge were always placed opposite the lamps, and no measurements were made on the corresponding parts of the prints.

\section{The Effect of the Wave-length of the Light}

The particular wedge examined by Toy \& Ghosh (I920) had a density at $300 \mathrm{~m} \mu$. which was $2 \cdot 2$ times the density at $420 \mathrm{~m} \mu$. Now the photographic emulsion is mainly affected by light of short wave-lengths, and it is thus clearly necessary to inquire whether it is appropriate to use the "visual" 
wedge constant when calculating the effective intensity of light falling on the photographic paper.

In the case of gaslight paper, it is possible to determine the "photographic" wedge constant directly. The paper is exposed behind the wedge on a number of occasions for the same duration, but at different distances from a point source of light. If precautions are taken to avoid stray light, the inverse square law can be applied, and the wedge constant determined by the inverse of the method used to determine the intensity of an unknown illumination, as described above.

An automobile head-lamp bulb was used as source of light, and exposures were made at distances varying from 50 to $400 \mathrm{~cm}$. The values obtained for the wedge constant were independent of the distance from the source of light, indicating the validity of the inverse square law, and the mean "photographic" value, so determined, was $38 \%$ higher than the "visual" value for parallel light as measured by a rectifier type photo-cell; the correction, therefore, is clearly of great importance.

Unfortunately, it is hardly possible to make similar direct measurements with "P.O.P.", owing to the difficulty of obtaining a point source of light sufficiently intense to allow of reasonable durations of exposure. An estimate of the probable magnitude of the correction, however, can be obtained from the spectral sensitivity curves of the emulsion before and after interposing the wedge between the source of light and the spectrograph; details of the methods used are given in a later section. The curves are shown in Fig. 2. If $A_{0}$ and $A_{w}$ are the areas below the curves obtained in the absence of the wedge, and in its presence, respectively, then it can be shown that $D^{\prime}=\log A_{0} / A_{w}$, where $D^{\prime}$ is the "photographic" density of the wedge at the point used. For "Slogas" the photographic density calculated in this way agreed within the limits of error with that determined directly. No very great accuracy can be expected, since the result depends considerably on the value chosen for the Schwarzschild constant.

It was not possible to obtain a spectral sensitivity curve for P.O.P. with the wedge in position owing to the very long exposures that would have been necessary. The curve can, however, be constructed, since the density of the wedge for any given wave-length is known from the observations on the gaslight paper. The photographic density is thus found to be $3 \%$ greater than the visual density, when tungsten filament light was used. For sunlight and daylight the corresponding corrections are 2 and $4 \%$ respectively.

\section{CHOICE OF EMULSION}

For general reasons, a relatively insensitive emulsion that requires the least amount of processing is desirable, and P.O.P. and gaslight paper appear to be the most suitable. A paper support for the emulsion also has some advantages over celluloid or glass. As there appear to be no records in the literature 
of measurements of the degree of failure of the reciprocity law in these emulsions, or of the spectral sensitivity of P.O.P., such measurements have had to be made.

\section{The Applicability of the Reciprocity Law to P.O.P. and Gaslight Paper}

The paper was exposed behind a neutral tint wedge to a uniform source of light for different times. In general, four exposures were made on each sheet of paper, of approximately I, 3, 8 and $24 \mathrm{hr}$. respectively. The prints were processed in the usual way, and dried. The method was sensibly the same as that described by Mallett (1922, 1923). Two millimetres were then trimmed off the sides of each wedge print, in order to eliminate the effects of shading by the binding of the wedge, and two of the four strips so formed were placed beneath a slit $2 \mathrm{~mm}$. wide in a piece of cardboard. One strip was then fixed so that the portion of the print beneath the slit was of a suitable density, and the other slid longitudinally until it also had the same density. Four such density matches were made, and then the two strips reversed in position, so as to eliminate as far as possible the effects of uneven illumination, accidental irregularities in the paper, and so forth, and four more matches made. The mean of the eight readings was then taken. If $l_{1}$ is the distance from the slit to the end of the print, corresponding to the zero end of the wedge, on the first strip, and $l_{2}$ the corresponding distance on the second strip, then, from the equation for the blackening of a photographic emulsion, given above,

$$
\log I-k l_{1}+p \log t_{1}=\log I+k l_{2}+p \log t_{2},
$$

where $I$ is the intensity of the illumination during the exposure. Hence

$$
p=\frac{k\left(l_{1}-l_{2}\right)}{\log \left(t_{1} / t_{2}\right)} .
$$

The comparison can, of course, be made at any desired density of the wedge prints, and, in general, four different densities were used whenever possible, defined arbitrarily as "very pale", "pale", "medium" and "dark". Such comparison at several different densities assists in eliminating the well-known unevenness in the response of different parts of the same emulsion. The use of a paper support, moreover, reduces this unevenness considerably, by allowing the processing solutions to reach the emulsion from both sides.

The Source of Light. It was necessary to obtain sensibly uniform illumination over a length of $10 \mathrm{~cm}$. (about $3 \mathrm{~mm}$. at each end of the wedge print were rejected in any case, owing to the shading by the binding which held the wedge between the glass plates), of sufficient intensity to produce an adequate density in the print in I hr., and free from random fluctuations over a period of at least $24 \mathrm{hr}$. Spatial uniformity was obtained in the case of gaslight paper by placing the wedge and paper a sufficient distance from the source of light, which was a I2 V. $36 \mathrm{~W}$. automobile head-lamp bulb. A distance of 
$2.5 \mathrm{~m}$. was found to give a suitable intensity. For P.O.P. a much greater intensity is required, and two such head-lamp bulbs were used, mounted about $10.5 \mathrm{~cm}$. apart, and with a sheet of surface flashed glass in contact with the bulbs. The wedge and paper were then mounted as close as was possible without introducing sensible non-uniformity of the illumination; this was about $7 \mathrm{~cm}$. from the lamp filaments. The uniformity of illumination was checked at intervals by means of a rectifier type photo-cell, and slight adjustments made if necessary. The two lamps usually had surprisingly equal emissions, but any small difference was eliminated by bringing one lamp closer to the wedge than the other.

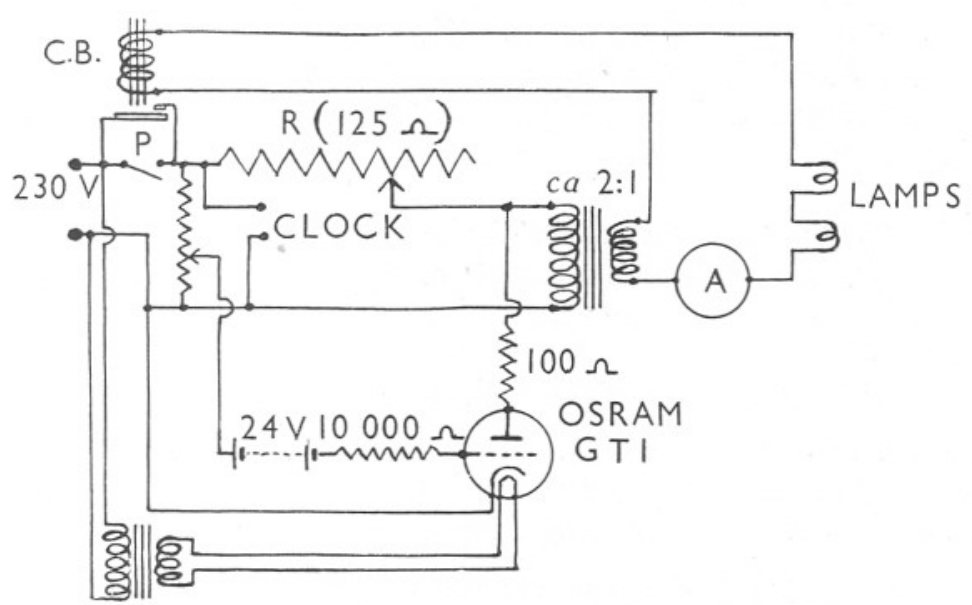

Fig. I. Diagram of circuit used for current stabilization in lamps used as source of illumination. $R$, main voltage dropping resistance; $P$, bell-push for starting current through lamps; $C B$, no-load circuit breaker.

Temporal uniformity was obtained by running the lamps off the A.C. mains in conjunction with one of two types of current stabilizer. The first involves the use of a gas-filled relay (or thyratron-for characteristics, see Hull, I929), and the circuit is shown in Fig. I. If the line volts rise, the negative bias on the grid of the relay falls more during each positive half-wave, and the current taken by the relay rises more than in proportion to the rise in the line volts. The potential drop across the resistance $R$ is increased, and the volts fed to the transformer, and hence the current in the lamps is reduced. A no-load circuit breaker was included as subsidiary equipment. If the current through the lamps fails, either through failure of the supply, or breakage of a filament, the circuit-breaker $C B$ opens, and protects the gas-filled relay from the rise of anode potential that would otherwise follow. The circuit-breaker cannot be closed again except by deliberately closing the bell-push $P$. The current regulation obtained was such that a $\mathrm{I} \%$ change in the lamp current was produced by a $5 \%$ change in the line volts; considerably better regulation was 
obtained for smaller changes in the line voltage. The G.T. I gas-filled relay does not take quite a large enough anode current for perfect regulation; in particular, occasional large mains fluctuations would extinguish it entirely. It would have been better to have included a step-up transformer in front of the circuit shown, so that a larger value of $R$ could have been used.

The second type of current stabilizer used depended upon the action of a barretter. Two type 304 Osram barretters were used in parallel, in the primary circuit of a Zenith regulating transformer, together with a variable resistance and the no-load circuit breaker, the lamps being connected in the secondary circuit. When two lamps were being used, it was necessary to step up the supply to about $350 \mathrm{~V}$., in order to bring the barretters to the proper operating conditions. The degree of regulation obtained was rather better than with the circuit shown in Fig. I for slow fluctuations, a I \% change in lamp current being produced by a $10 \%$ change in line voltage. Owing to the slow response of the barretters, however, they were unable to take charge of rapid fluctuations.

In both circuits, an electric clock was connected in the circuit beyond the no-load circuit-breaker. This indicated the duration of the exposure in the event of the circuit-breaker opening for any reason.

\section{The Value of the Schwarzschild Constant}

A. P.O.P. (Ilford matt white). Seventeen experiments were performed involving sixty-eight exposures and I24 determinations of the Schwarzschild constant. The results are shown in Table III. Previous to these, ten experi-

Table III. The Schwarzschild Constant for P.O.P.

Duration of
exposure,
hr.
I-3
$3-8$
$8-24$

\begin{tabular}{|c|c|c|c|}
\hline \multicolumn{4}{|c|}{ Density of print } \\
\hline Very pale & Pale & Medium & Dark \\
\hline$I .05 \pm 0.023$ & $I .02 \pm 0.013$ & $I \cdot 04 \pm 0.012$ & - \\
\hline $1.04 \pm 0.033$ & $\mathrm{I} .03 \pm 0.022$ & $I .00 \pm 0.020$ & $0.83 \pm 0.057$ \\
\hline $1.02 \pm 0.015$ & $I .05 \pm 0.018$ & $1.07 \pm 0.010$ & $\mathrm{I} .01 \pm 0.025$ \\
\hline
\end{tabular}

ments had been performed while the technique was being developed. The results of these were more erratic, but the general conclusions to be drawn are the same.

Three general conclusions can be drawn. (I) The value of the Schwarzschild constant is independent of the duration of the exposure up to $24 \mathrm{hr}$. (2) The value of this constant is independent of the density of the print, except in the case of the exposures of medium duration, where there is a definite fall at high densities. It should be pointed out that in the limit, of course, the Schwarzschild constant must fall to 0 as the density increases, since there is a definite limit to the density which can be produced by any amount of illumination. The densities used were all well below this limit. (3) The departure from 
the reciprocity law is barely significant. In addition, it was shown that the value of the Schwarzschild constant is unaffected by changes, over wide limits, in the duration of fixation, or in the concentration or temperature of the hypo used. A few not very accurate measurements were made on unfixed prints; in these, the value of the constant was about $I \cdot 5$.

The value of the standard deviation indicates an uncertainty of $\pm 7.5 \%$ for a single measurement. This includes the uncertainty in the constancy of the illumination in two experiments, as well as the uncertainty in the matching of the densities, and the lack of uniformity of the emulsion, but does not include the uncertainty in the value of the wedge constant. The effect of this will become proportionately greater as the total illumination measured becomes greater. It may be expected, therefore, that the uncertainty in the determination of an unknown illumination will be between \pm 5 and $\pm 10 \%$.

B. Gaslight paper (Illingworth Slogas matt soft). A smaller number of determinations was made with gaslight paper, as it very soon became apparent that this emulsion is not suited for photometric work involving long durations

\section{Table IV. The Schwarzschild Constant for Gaslight Paper. (MEAN OF ALL PRINT DENSITIES)}

Duration of exposure, hr.

$\begin{array}{cccc}3 / 4 & 3 / 4-3 & 3-15 & 15-30 \\ 0.94 \pm 0.01 & 0.88 \pm 0.02 & 0.80 \pm 0.02 & 0.8 I \pm 0.03\end{array}$

of exposure. The value of the Schwarzschild constant as shown in Table IV is considerably less than I and appears to fall with increase in the duration of the exposure.

Jones \& Huse (1923) give values of the Schwarzschild constant for three types of emulsion; at very low intensities (exposures lasting up to a few hours) they find values of 0.63 for a high speed emulsion, 0.88 for a medium speed emulsion, and 0.68 for a low-speed emulsion. In all cases the value of $p$ fell as the intensity of illumination was reduced. Baker (I924), working on three brands of panchromatic emulsion and with exposure times up to $200 \mathrm{sec}$., reports values of $0.80-0.93$. Again, the value fell as the exposure time was increased.

The Spectral Sensitivity of the Emulsions. In all photometric methods it is important to know at least approximately the limits of wave-length to which the method is sensitive. For biological work, moreover, it is desirable that these limits should at least be within the visible spectrum. Spectral sensitivity curves of various photographic emulsions to tungsten filament light are given by Davis \& Walters (1922). These authors find that ordinary plates have a maximum sensitivity to light of wave-length between 450 and $475 \mathrm{~m} \mu$., while the slower transparency and lantern plates have a maximum sensitivity in the region $420-460 \mathrm{~m} \mu$. These curves, however, give little indication as to the spectral sensitivity to be expected from an emulsion such as P.O.P. which is not developed. 
Accordingly, fresh observations were made with a Hilger quartz spectrograph, using an automobile head-lamp bulb as source of light; under the conditions of operation, the colour temperature would have been about $2800^{\circ} \mathrm{K}$.; a slit width of $2 \mathrm{~mm}$. had to be used to get a sufficiently high illumination to affect P.O.P. in a reasonable time. The approximate sensitivity curves shown in Fig. 2 were obtained by making a series of exposures of different durations and plotting the wave-length at which a standard amount of blackening had been produced, against $\mathrm{I} / t^{p}$, where $p$ is the Schwarzschild

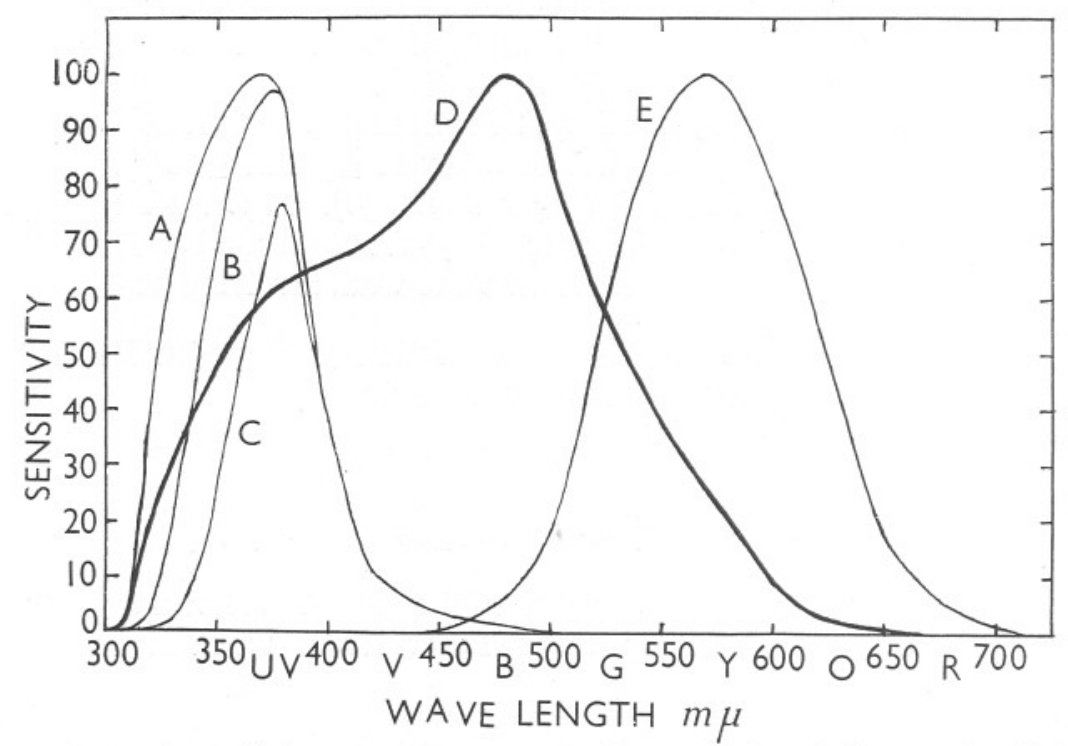

Fig. 2. Spectral sensitivity curves to tungsten filament light. Ordinates: Sensitivity in arbitrary units. Abscissae: Wave-length. (A) Illingworth Slogas. (B) The same with neutral tint wedge (zero end) in front of slit, i.e. showing the effect of absorption by the glass mounts. (C) The same with neutral tint wedge (density $=\mathrm{I} \cdot \mathrm{O}$ ) in front of slit, the ordinates being multiplied by IO. (D) Ilford P.O.P. (E) The human eye. Curve $E$ is derived from the luminosity figures of Gibson \& Tyndall (I923) and the distribution of energy with wave-length in tungsten filament light given by Priest (I922).

constant for the emulsion in use. Each ordinate was then corrected for the varying dispersion of the spectrograph and the varying obliquity of the light falling on the paper.

The curve for gaslight paper differs very markedly from that given by Davis \& Walters for ordinary photographic plates, the maximum sensitivity being in the neighbourhood of $370 \mathrm{~m} \mu$. As a check, a series of exposures was made on an Imperial S.R. plate; the maximum sensitivity was found to be around $465 \mathrm{~m} \mu$., in good agreement with the observation of Davis \& Walters. The shift of the maximum sensitivity towards the shorter wave-lengths in the case of gaslight paper is in agreement with the similar but smaller shift observed by Davis \& Walters in the case of transparency and lantern slide plates. 
The behaviour of P.O.P. is very different. If the unfixed print is examined (Fig. 3) it will be seen that the blackening begins at a wave-length of about $370 \mathrm{~m} \mu$. The density, however, does not increase at all rapidly in this region, but extends toward the red end of the spectrum, the maximum density after $32 \mathrm{hr}$. exposure being produced at about $520 \mathrm{~m} \mu$. It is to be supposed that the surface layers of the emulsion become blackened first, and that these then absorb the shorter wave-lengths more than the longer, and so prevent the former from penetrating to the deeper layers. On fixing, the density of the

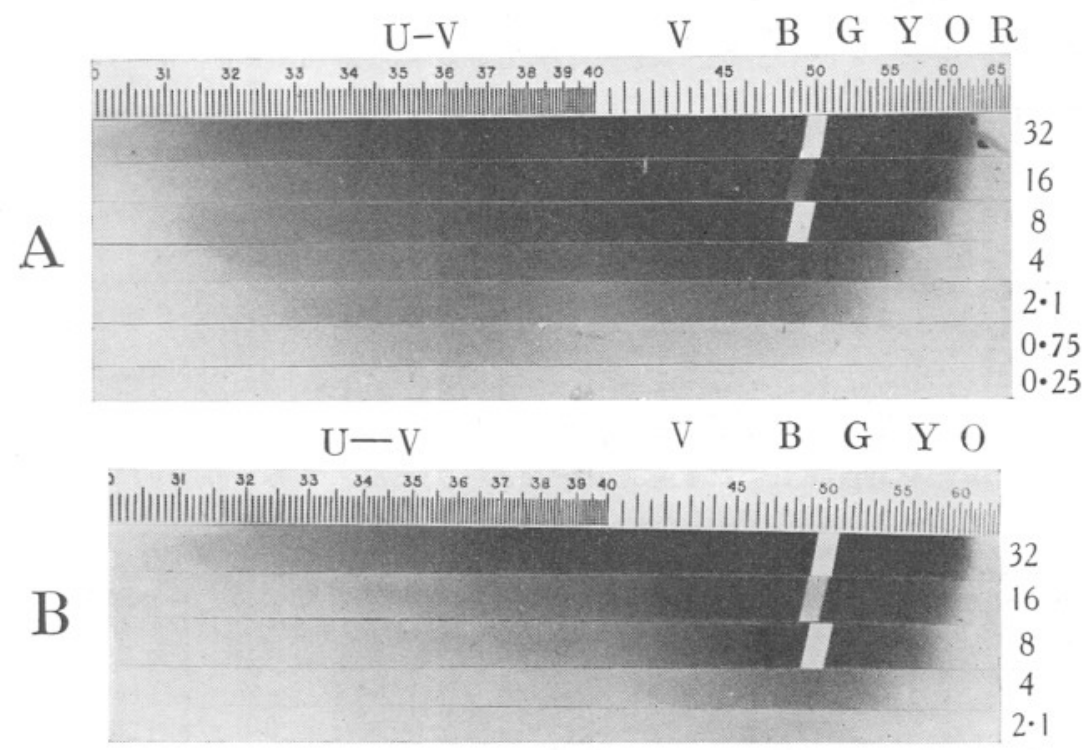

Fig. 3. Photographs of continuous spectrum of tungsten filament light taken on P.O.P. (A) Unfixed. (B) Fixed. The duration of each exposure is given in hours. The white mark across the upper three exposures in each case is due to the glass plate which was placed in front of the paper cracking during the $16 \mathrm{hr}$. exposure; this was made before the $8 \mathrm{hr}$. exposure.

whole print is reduced, and the weak effects of the short wave-lengths in the short exposures are removed entirely. The most effective wave-length for the fixed print, i.e. that at which blackening is first produced, is thus shifted to about $485 \mathrm{~m} \mu$. Introduction of the dispersion correction, shifts this maximum to $480 \mathrm{~m} \mu$.

It is clear, therefore, that for purposes of photometry in visible light, P.O.P. is greatly to be preferred to gaslight paper, or, in all probability, to any emulsion after development. Not only is it sensitive to a wider range of wave-lengths, but it has the added advantage of obeying the reciprocity law within the limits of experimental error. 


\section{Standardization and Calculation of the Results}

A I2 V., $36 \mathrm{~W}$. automobile head-lamp bulb was used as standardizing light, operated from one of the current stabilizing circuits already described. An elaborate circuit of this kind is not necessary, as the exposure is only of a few hours' duration, and the current can easily be regulated by hand. The paper was exposed to this light at a distance of $30 \mathrm{~cm}$., a sheet of flashed glass being placed in contact with the lamp bulb. Three exposures were usually made for about 2, 4 and $6 \mathrm{hr}$. respectively, giving three patches of different density. The wedge print was then compared with each of these patches in turn exactly as described above for the determination of the Schwarzschild constant. The mean of the three results was then taken. The method of calculation is indicated by the following example.

An exposure was made on the roof of the Plymouth laboratory from 20.30 G.M.T. I2 July I934 to 20.30 I3 July, say I7 hr. of daylight. The weather was mainly overcast. No diffusing screen was used and consequently the exact value of the wedge constant to be used is a little uncertain. The results are given in Table $\mathrm{V}$, in terms of the intensity of the standardizing light. If

Table V. Analysis of a Typical Record

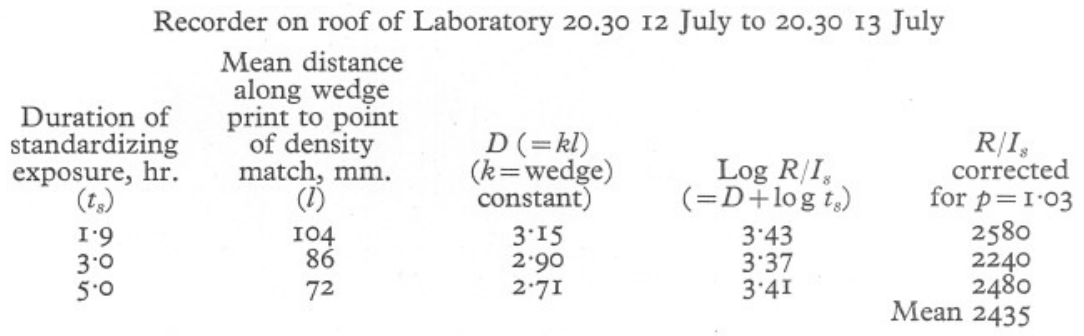

this is measured in visual units, then the unknown illumination can also be directly expressed in visual units, provided that the distribution of energy with wave-length is the same in the unknown as in the standardizing source of light. For other sources of light-notably daylight-correction factors are necessary. The magnitude of these colour correction factors are subject to very considerable uncertainty since the methods used in their derivation fail to take more than an approximate account of the behaviour of photographic emulsions in lights of different intensities and different wave-lengths. Measurements made in any one quality of light, however, will be consistent among themselves even though their absolute magnitudes in visual or energy units are uncertain. The object of the standardizing exposure is primarily to eliminate random differences between one sheet of paper and another, arising from inherent differences of sensitivity, or from small differences in processing.

These correction factors express the relative brightness of the two sources 
of light as measured by (I) the human eye, and (2) the photographic emulsion. The relative brightness to the human eye on the one hand, and to the photographic emulsion on the other hand, of tungsten filament light can be discovered from a knowledge of (a) the luminosity curve of a constant energy spectrum (Gibson \& Tyndall, I923), (b) the distribution of energy with wave-length in the tungsten filament light (Priest, 1922), and (c) the spectral sensitivity curve of the photographic emulsion to tungsten filament light, described in the previous section. The ratio between the area below the luminosity curve for tungsten filament light and that below the photographic spectral sensitivity curve for the same light, gives us the photographic effectiveness of each lux of this light; we will denote this by $E_{w}$. For daylight, a similar factor can be derived from $(a)$ the luminosity curve as above, $(b)$ the distribution of energy with wave-length in daylight (Davis \& Gibson, I93I), and $(c)$ the spectral sensitivity curve of the photographic emulsion to daylight, derived from the similar curve for tungsten filament light by means of the two energy distribution curves; we will denote this factor by $E_{d}$.

The figures used for daylight are those given by Davis \& Gibson for the distribution of energy with wave-length in the sun's radiation outside the earth's atmosphere. These authors give reasons for believing that the colour temperature of an overcast sky is very closely equal to that of the sun outside the earth's atmosphere.

The figures given by Priest (I922) for the energy distribution with wave-length of a tungsten filament lamp, are for a lamp with an efficiency of 15.6 lumens per watt. The distribution of radiant energy in the plane of the filament used under normal operating conditions (II.6 V., $2.8 \mathrm{amp}$.) was measured approximately by means of a photo-cell, and the luminous efficiency calculated to be about I6 lumens per watt, a sufficiently close approximation for the present purposes.

The illumination on the photographic paper when exposed to the standardizing light was measured by means of a Holophane Lumeter, and found to be 550 lux. The value of $E_{w}$ was found to be 0.26, so that the "photographic" intensity of the standardizing light was $550 \times 0.26=\mathrm{I} 43$ "photographic units". Similarly, the value of $E_{d}$ was found to be 0.69 , so that if we have a daylight illumination which is, photographically, $A$ times the standard of I43 photographic units, its visual intensity will be $\frac{\mathrm{I} 43 \times A}{0.69}$. Thus in the example given in Table $\mathrm{V}$, the total vertical illumination received becomes $2435 \times \frac{\mathrm{I} 43}{0.69}=505$ kilolux-hours. The recorder, in this experiment was placed on a horizontal surface, and so the readings represent the vertical component only of the total illumination. There is no reason why a photographic recorder of this type should not be placed within a globe integrator, such as is described by Atkins \& Poole (1936a), in which case the total illumination from all directions would be measured.

The question now arises as to the appropriate value to be used for the factor $E_{d}$ during bright sunshine. Owing to the scattering in the upper 
atmosphere, sunlight at the earth's surface is relatively deficient in the blue end of the spectrum, the scattered light being apparent in the blueness of the clear sky. If the recorder is placed in a spherical integrator, so that it receives the whole radiation from the celestial hemisphere, the direct sunlight and the scattered skylight would be recombined, and the value 0.69 would still be appropriate for the factor $E_{a}$. When used to record the vertical component only, it will receive one-half of the scattered skylight, and a fraction of the direct sunlight depending on the altitude of the sun. From the figures given by Davis \& Gibson (I93I), it can be calculated that the photographic effectiveness (P.O.P.) of each lux of mean noon sunlight is 0.46 , and the calculated values of $E_{d}$ for various altitudes of the sun are given in Table VI. The low

\section{Table VI. The Photographic Effectiveness of Daylight + Sunlight FOR P.O.P.

$\begin{array}{lccccc}\text { Altitude of sun } & 75^{\circ} & 50^{\circ} & 38^{\circ} & 30^{\circ} & 10^{\circ} \text { and less } \\ \text { Value of } E_{d} & 0.62 & 0.64 & 0.665 & 0.69 & 0.60 \text { and less } \\ & \text { * According to amount of mist and cloud. }\end{array}$

value for very low altitudes is only estimated; when the sun is very low, there will be a considerable amount of scattering by the thick layer of atmosphere, and the sunlight will be distinctly yellow. Moreover, this yellow light will be reflected by the under surfaces of clouds and mist, and so will not be compensated by the blueness of the sky. In view of the very great variability of light conditions at sunrise and sunset, it would be unwise to place any reliance on photographic observations made at these times.

It should, perhaps, be pointed out, to avoid misconceptions, that we are not concerned, here, with the relative intensity of the sunlight and the skylight. As is shown by the figures given by Atkins \& Poole $(1936 b)$, this quantity varies very greatly according to the altitude of the sun, the time of year and type of sky. We are concerned only with the apparent colour temperature of the light falling on the photographic recorder. Two extreme examples will illustrate the difference between these two quantities. (I) Outside the earth's atmosphere, there is no skylight (to all intents and purposes), and the intensity ratio sun/sky is infinity. (3) When the sky is completely overcast, there is no sunlight, and the intensity ratio is zero. Yet the colour temperature in both cases is the same.

\section{USE OF THE METHOD IN THE FIELD}

The detailed technique must depend upon the exact nature of the measurements to be made, and the only essentials are some means of clamping the wedge in contact with the sensitive paper, and suitable protection from the weather. That part of the paper which is not underneath the wedge must be protected from the light, so that the standardizing exposure or exposures may be made on the same sheet. There is no necessity to perform the standardization in the field. The prints, if reasonably cool and dry, can be kept in the dark without deterioration certainly for a week, and probably for 
longer, before fixing. When used on land, and in bright sunlight, the recorder should be placed as far as possible so that the direction of the sun is at right angles to the long axis of the wedge; the small errors due to the parallax between the wedge and the emulsion are thus eliminated.

\section{SUMMARY}

I. The photographic neutral-tint wedge method of measuring the total illumination falling on an area over a long period of time is described.

2. A study is made of the chief sources of error inherent in photographic methods, i.e. (a) the uncertainty in the value of the wedge constant, and (b) the failure of the reciprocity law, in that the amount of blackening of the photographic emulsion is not, in general, strictly proportional to the product of the intensity of the illumination and the duration of the exposure.

3. It is shown that P.O.P. obeys the reciprocity law sufficiently closely for durations of exposure between I and $24 \mathrm{hr}$. Gaslight paper, on the other hand, can only be used when the duration of exposure is less than about I hr.

4. It is shown that when P.O.P. is used, and a sheet of diffusing glass is placed above the wedge, the value of the wedge constant is sensibly independent of the direction and quality of the incident light.

5. Spectral sensitivity curves to tungsten filament light for gaslight paper and P.O.P. have been evaluated. From these, and other data, correction factors have been calculated for converting the photographic measurements of illumination into visual units.

6. It is concluded that the method is sufficiently reliable for use in the field, where its simplicity and the small amount of apparatus required, make it specially suitable. The experimental uncertainty is about \pm 5 to $\pm 10 \%$.

\section{REFERENCES}

Atkins, W. R. G. \& Poole, H. H., I936a. The photo-electric measurement of diurnal and seasonal variations in daylight, and a globe integrating photometer. Phil. Trans. Roy. Soc. A, Vol. 235, p. 245.

_ - I936 b. Photo-electric measurements of the luminous efficiency of daylight. Proc. Roy. Soc. B, Vol. I2I, p. I.

BAKER, E. A., I924. The law of blackening of the photographic plate at low densities. Proc. Roy. Soc. Edin. Vol. xLv, p. I66.

Benson, D. E., Ferguson, W. B. \& Renwick, F. F., I9I8. A convenient and accurate photometer for the measurement of photographic densities. Photogr. Fourn. Vol. 58, p. 155 .

Cooper, J. O. \& SAyce, L. A., I932. A photographic daylight recorder. Fourn. Sci. Inst. Vol. 9, p. 282.

Davis, R. \& Gibson, K. S., I93I. Filters for the reproduction of sunlight and daylight and the determination of colour temperature. Bur. Stand. U.S.A. Misc. Publ. No. II4.

DAVIS, R. \& WALTERS, F. M., I922. Sensitometry of photographic emulsions and a survey of the characteristics of plates of American manufacture. Bur. Stand. U.S.A. Sci. Papers, Vol. 18, p. r. 
Dobson, G. M. B., Griffith, I. O. \& Harrison, D. N., I926. Photographic Photometry. Oxford, University Press.

Eder, J. M., 1919. Ein neues Graukeilphotometer. Photogr. Korr. Bd. 56, p. 244. $1920 a$. Ein neues Graukeilphotometer. Photogr. Korr. Bd. 57, pp. I, 4I, 83, 304.

- I920b. Ein neues Graukeilphotometer. Halle, Wilhelm Knapp.

Gibson, K. S. \& Tyndall, E. P. T., 1923. Visibility of radiant energy. Bur. Stand. U.S.A. Sci. Papers, Vol. I9, p. I3I. (Quoted by Ives, H. E., Int. Crit. Tables, Vol. 5, p. 436.)

GolDBERG, E., I9I0. The preparation of prismatic wedges of neutral colour for photometric work. Brit. Fourn. Photogr. Vol. 57, p. 648.

GRUBER, M., I924. Über ein Methode zur Messung des Lichtgefälles im Wasser mit Hilfe des Eder-Hechtschen Graukeils. Intern. Rev. f. Hydrobiol. Bd. I2, p. I7.

HeCht, W., I9I8. Das Graukeilphotometer im Dienste der Pflanzenkultur. S.-B. Akad. Wiss. Wien. II $a$, Bd. I27, p. 2283.

Hull, A. W., 1929. Hot cathode thyratrons. Gen. Elect. Rev. Vol. 32, pp. 313, 390.

JONES, L. A. \& HUSE, E., I923. On the relation between time and intensity in photographic exposure. Fourn. Opt. Soc. Amer. Vol. 7, p. r079.

MaLlett, R. A., 1922. On the failure of the reciprocity law in photography. Phil. Mag. Vol. 44, p. 904.

- 1923. On the failure of the reciprocity law in photography. Phil. Mag. Vol. 45, p. 800 .

OBERDORFER, E., I928. Ein neuer Apparat zur Lichtmessung unter Wasser. Arch.f. Hydrobiol. Bd. 20, p. I34.

PRIEST, I. G., I922. Colour temperature of high-efficiency sources by the rotatory dispersion method. Fourn. Opt. Soc. Amer. Vol. 6, p. 27. (Quoted by Coblentz, W. W., Int. Crit. Tables, Vol. 5, p. 245.)

SCHWARZSCHILD, K., I900. On the deviations from the law of reciprocity for bromide of silver gelatine. Astrophys. Fourn. Vol. II, p. 89.

Toy, F. C. \& GHosh, J. C., I920. The absorption of light by the Goldberg wedge. Phil. Mag. Vol. 40, p. 775. 